\title{
An approach to knowledge representation and performance measurement for a Quality Engineering System
}

\author{
J. A. Heredia*, I.-S. Fan**, F. Romero* , P. Lowenthal** \\ *Departamento de Tecnologia. Universitat Jaume I. Campus Penyeta \\ Roja, 12071 Castellon. Spain. \\ Tel.34-64-345680Fax.34-64-345646e-mail: heredia@tec.uji.es \\ **The CIM Institute. Cranfield University. United Kingdom.
}

\begin{abstract}
This paper presents a methodology which can assist an organisation to identify and to represent the knowledge and information required by a Quality Engineering System. The structure of integrated knowledge representation for product and processes to support Quality Engineering is described. Integrated object oriented modelling and simulation is identified as a valuable tool for knowledge management. The relationship between Quality Engineering and the Performance Measures of Quality Loss, Cycle Time and Production Cost is explained. The continuous management of knowledge and information, recording, filtering and applying the lessons learnt leads to an increase in manufacturing performance both in the long term and short term.
\end{abstract}

\section{INTRODUCTION}

The Quality System conceived by Taguchi is an integrated system in which all the company activities are involved to improve business performance: "Ideally, an integrated system of overall quality control, in which all activities interact to produce products with minimum deviations from target values, will minimise quality costs and make the most economic use of human and other company resources" (Taguchi, 1989).

Within the domain of manufacturing the more commonly accepted competitive dimensions which lead to measures of manufacturing performance are Quality, Time and Cost. The Quality Engineering System we envisage consist of a set of integrated techniques and methods that support Quality Control and Maintenance activities both on-line and off-line. The aim is to improve manufacturing performance in these three ways: decreasing production costs, cycle time and providing the desired quality to the customer. An important element in achieving superior manufacturing performance is the requirement for the systematic use of knowledge available within an organisation and a structured practice of acquiring and generating new knowledge.

As Zeleny (Zeleny, 1989) states, "knowledge has become a primary form of capital" . Although knowledge is often the most important form of capital, it is generally hidden under the concept of intangible assets and is poorly managed. This occurs despite the 
evidence that organisations prosper because of their know-how, and that they use this know-how as a primary driver for their business and operations strategies (Anderson, 1991). Traditionally, when a company faces increased competitive pressure, it makes more effective use of its physical resources in order to survival. Today companies need to treat knowledge as a key resource and make more efficient use of it. Knowledge, like power, increases when it is communicated and can only be effective when it is explicit.

Knowledge must be shared at all levels in an organisation for effective continuous improvement, "Hard work and best efforts, put forth without guidance of profound knowledge, may be well at the root of our ruination. There is no substitute for knowledge" (Deming, 1989). In essence the process of improvement is described by the framework of the "scientific method". In terms of quality improvement, the scientific method is known as the Shewhart's cycle, or the PDSA (Plan, Do, Study, Act) (Shewhart, 1939). Continual improvement of product and process demands that the knowledge generated from each previous application of the PDSA cycle be synthesised and recorded to drive the strategy and implementation of the next PDSA cycle.

\section{KNOWLEDGE REQUIREMENTS}

In order to achieve improvement, methods, procedures and physical channels need to be established for the acquisition and dissemination of knowledge. This structure facilitates the use of information and subsequent generation of knowledge. A common knowledge and information base will support the learning process within an organisation.

Therefore the natural question is : Which kinds of Quality Engineering knowledge are needed to improve measures of manufacturing performance?_The answer includes that kinds of knowledge that permit us to understand the mechanisms that allow the company to: implement an effective maintenance strategy, increase equipment availability; improve the equipment capacity; make the processes and products more robust; use effective automatic diagnosis tools; adjust the processes when required; select the most efficient inspection plan; reduce the cycle time; select the new equipment most efficient.

A more systematic way to identify the knowledge requirements can be achieved by listing and analysing the kinds of knowledge and information related with Quality Engineering that can be useful for each of the manufacturing activities: Product Planning, Product Design, Process Design, Production and After Sale Service. This permits us to detect a suitable knowledge representation and its relationships.

\section{a) Product Planning}

In the product planning stage the competitiveness of a manufacturing enterprise depends on its ability to design and develop products that can achieve the customer's desired goals (Edmondson, 1992), while keeping production cost below those of its competitors. In the product planning stage the function, price and operating life of new products are decided (Taguchi, 1993). Therefore, in the product planning phase it is necessary to use knowledge 
about customer needs and wants together with a correct translation of these requirements into detailed product features, and knowledge about the competitive products. This identifies a type of knowledge which we term behavioural knowledge (see, page 5) and can be expressed using the first matrix within the QFD methodology.

\section{b) Product and Process Design}

The next step involves translating the technical requirements into target values associated with the product's quality characteristics and to simultaneously design the process that will achieve these targets at the lowest cost. This is the responsibility of the product-process designers. Both product and process design can be divided into five stages (Taguchi, 1989):

1. System selection: Firstly, one must consider possible systems that can perform the required functionality for the product and process. Consequently knowledge regarding possible attributes for the product and process characteristics are required. This is the domain specific knowledge or technological knowledge that encompasses the theoretical and experiential knowledge about the structures, functions and behaviour of the process and products. This knowledge can be represented as analytical equations or as rules. A good foundation in theoretical knowledge is a prerequisite for new systems development. The system selected can be a redesign or improvement of an existing one. A detailed process model is very useful for process improvements ( including equipment improvements), especially for time improvements ( some people call this Re-engineering). Therefore knowledge is needed about the performance of the different possible systems. All systems can be viewed as a set of functions in a logical sequence supported by a structure of mechanisms that behave in different ways under different circumstances. To evaluate the behaviour of a system before implementation it is necessary to model the system. A modelling software tool can be used to capture the structural, functional and behavioural knowledge.

2. Parameter design: At this stage, the design engineers specify the nominal levels for the products and process attributes (design parameters) of the chosen system. These levels are optimised to improve quality and reduce cost. In order to conduct efficient parameter design it is necessary to use optimisation methods: experimental methods, response surface, Linear and Dynamic Programming. The results of applying these methods should be target values for the products and process attributes and equations describing the relations between the response and the control variables. Furthermore the knowledge generated by conducting off-line and on-line experiments has to be recorded so that it can be used for successive design processes showing the causes, modes and effects of failures for the different product's components and process variables.

3. Tolerance design and specification: The objective of this phase is to decide the required trade-off between tolerances and cost for the system that is being designed. The objective evaluation criteria used by Taguchi are the quality loss of products. 
After tolerance design the grades of materials and the tolerance limits of components must be specified and contracts with material and component suppliers must be signed. This requires documented information regarding a suppliers capabilities and quality practices.

4. Quality management for the production process : At this stage, feedforward and feedback control systems must be planned to control the statistical distribution of the key quality characteristics and the equipment availability. To get the full potential that the Quality Engineering methods and Information Technology offers we have to :

- Select the best Quality Control and Maintenance Plans from several possible alternatives by evaluating the performance measures which provide when translated into financial terms, the greatest savings and benefits. This plan has to include detailed instructions to gather and manage data from the shop-floor.

- Design the diagnostic process to locate the root cause and a corrective action process to eliminate it.

Methods and techniques from Automatic Control Engineering Systems, Statistical Process Control, Measurement and knowledge of the current state of the production system in terms of stability and capability are required to design this control system.

\section{c) Production}

Once the product and process have been designed and developed production starts and the on-line quality control and maintenance methods have to be applied. Periodically, the quality of the products and the process have to be measured while production is taking place, at this time the causes of defective products and processes should be rooted out, and the production process readjusted to the intended operational conditions. The two objectives for using the knowledge mentioned above are:

- to find the root causes for the variation from the target values; and

- to detect the need for acquiring new knowledge about the process behaviour.

\section{d) After Sales Service}

Once a product is in the market place, feedback of a product's behaviour in the field is required. 


\section{KNOWLEDGE REPRESENTATION}

After the knowledge requirements identification the next logical step is to consider how this knowledge will be represented. A Quality Engineering System must include qualitative analysis tools to capture and systematically use the experience and judgement of the organisation, as well as quantitative tools requiring relevant process data. Experience and judgement are more critical when the tools are used in a proactive mode ( problem prevention) rather than in a reactive mode. A proactive strategy requires an emphasis on physical cause-effect knowledge. A reactive strategy emphasises loss accounting and data intensive statistical inferences to justify action. "Each organisation must assess the tradeoff between proactive and reactive strategies which makes technical and economic sense to it and is compatible with the knowledge and experience of its people" (Kolarik, 1995).

In both strategies knowledge representation is a key issue for planning and inferencing. "Good knowledge representation should neither lose any useful knowledge nor create redundancies, enabling knowledge transformation, abstraction, sharing, and inferencing to occur accurately and efficiently" (Shewhart,1939). An important factor in process knowledge representation is the use of Operational definitions. Operational definitions have two components: objects and attributes (Deming, 1986). Based on these two components and as a consequence of the analysis that has been conducted in the previous section, three basic knowledge classes can be identified that cover all the manufacturing functions. They are Structural, Functional and Behavioural (Liu, X, 1992):

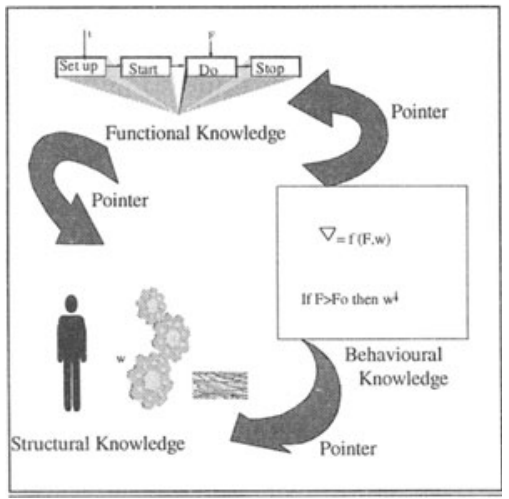

Figure 1. Knowledge Representation Illustration

Structural knowledge: represent durable and convertible resources like equipment, people, environmental variables and products. Structural knowledge representation includes two things which describe the features of process components: component attributes and physical connections with other components. The attributes and connections represent the components characteristics and position. 
Functional knowledge: describes the manufacturing and product operations in a logical sequence. The operation attributes are the process variables. A pointer from the functional representation to the structural representation extends the search ability (within the diagnostic module) which can go from activities to constitutive elements.

Behavioural knowledge: represents the relationship between attributes of the structural and the functional representations. Typically, this relationship can be expressed in the following forms: QFD weights, FMEA, Causal Rules, Analytical Models and Simulation Models. These knowledge representations have to be compatible and coherent. The pointers to the structural and the functional representations close the knowledge integration loop.

To be effective the Quality Engineering System that is being developed by the authors incorporates, as an important part, a modelling tool capable of capturing the structural, functional and behavioural knowledge representations. A basic requirement is the capability for hierarchical decomposition, that will permit the analysis in an undefined number of layers. Other important requirements are customisation of objects and freedom to programme the behaviour and relationships of the objects through methods. With such a software tool knowledge modelling and maintenance is facilitated. One such tool used by the authors is Simple++ (Simple++,1994). Simple ++ is a modelling and simulation software tool object oriented. Figure 2 illustrates how this tool can be used to represent and maintain knowledge. This picture show a typical screen in the process of modelling a discrete piece part manufacturing plant.

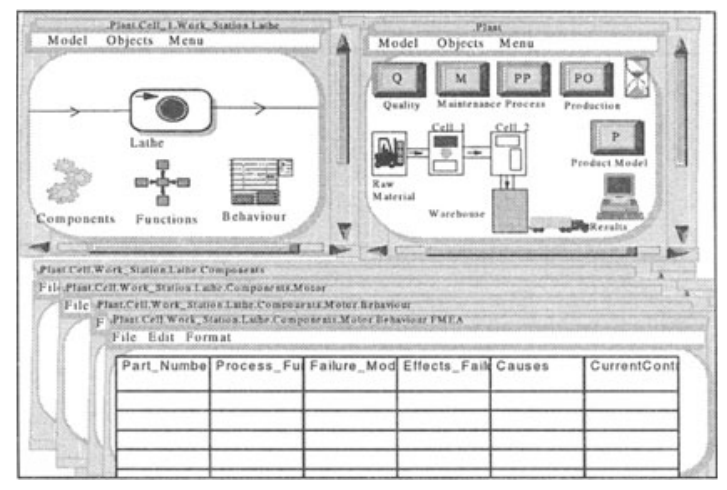

Figure 2. A typical screen in the process of modelling using Simple ++

The user beginning from the highest simulation level of the plant (right-up window) has selected Cell_1. After clicking on the lathe in this cell (left-up window), he has been navigating through the lathe structure and located the lathe motor. Once this has been done he has selected the icon that open the motor behaviour window, and finally the FMEA option. At this moment the user is editing the FMEA table. This behavioural knowledge will be translated in rules and exported to the knowledge base of the Expert System that will be used to assist in the diagnostic of root causes when and out of control signal will be detected. 


\section{MANUFACTURING PERFORMANCE MEASURES}

The goals of Quality Engineering ( reflected by the Taguchi Paradigm) are to increase the value of the product to the customers, to reduce the total production cost, and to reduce the time required for all activities within the organisation. Quality Engineering methods influence these three basic performance metrics directly. In addition there is a synergistic effect between these performance measures.

With simulation modelling an entire manufacturing system ( processes, equipment and information models) can be modelled. Through the data exchange interface actual company data can be used to conduct a simulation. This integrated model and simulation will support the Quality Plans selection, as well as the evaluation of effects of these Plans on the Performance Metrics.

\subsection{Quality Engineering System and Time}

Quality Engineering methods can improve both the average and the variance cycles time for each process step.

By using Quality Engineering methods the average time can be reduced continuously aiming towards the goal time. We define the goal time for a process as the minimum feasible time required to perform the desired activity with the available technology, namely the time in which it is required to add value to the product plus the minimum necessary time due to the technological limitations. For example a typical machine tool will have a goal time equal to the service rate plus the minimum theoretical time needed for set-up. To set the goal time it is necessary to (1) analyse the process in detail and detect any operations that add no value, and/or (2) use data from benchmarking practice, and/or (3) redesign the process using the model that would have been generated during the system design.

Considering the design process "efficient methods of optimisation and variation reduction can shorten development time. They reduce the amount of experimentation necessary to achieve the desired results. However, even more important, their proper use early in the design stage will prevent problems early on. This reduces the number of problems and delays that develop later on which typically delay market entry"[Taylor,1991].

Also, the goal time could be affected directly because the Quality Engineering System supports the recording and accumulation of knowledge that can lead to breakthroughs in technology development.

During Production the time variance used to perform an activity will be reduced because of the implementation of "optimum" maintenance and quality control plans that leads to: less machine break downs, less variation in the physical quality characteristics in the products, shorter diagnostics and recovery intervals, clearer operations instructions, and improved resources availability assisting to achieve better schedules. 
The direct consequences from decreasing the cycles times in a manufacturing process is to increase flexibility and to reduce the work in process and inventory costs. A simulation model can be thought as a function that turns input parameters into output performance measures. The explicit form of this function, for a concrete manufacturing system, can be approximated using a regression model, where the independent variables are the simulation input parameters,- in this case the different cycles times and production planning strategies and the response variable is the response of interest - in this case, for instance the inventory costs-. In order to obtain the data and fit the regression model a factorial design can be performed running several simulation replications.

\subsection{Quality Engineering System and Quality Loss}

We assess the quality of the products and process using the Taguchi Loss Function. The Quality Loss Function is the additional cost that the customer has to pay, (in addition to production costs that includes the traditional Quality Costs), because the goal nominal values for the designed quality characteristics have not been achieved [Taguchi, 1986].

Naturally, the basic objective in Quality Engineering is to reduce this Loss using the off-line and the on-line well known methods for Optimisation and Variation Reduction [Taylor, 1991] and through effective Preventive Maintenance [Nakajima, 1988].

If we consider that in each process operation we are going to measure several key quality characteristics, then the function loss for the whole process can be estimated by the addition of the individual functions. This aggregate loss function provides an index of the total quality of the process because it takes into consideration all the key functional characteristics.

\subsection{Quality Engineering System and Production Costs}

Quality Engineering methods directly influence Productions Costs by decreasing Quality Costs and Maintenance Costs. In addition, "less expensive manufacturing processes may be used by making the product robust to the process" and "less expensive materials can be used by making the product robust to material variations"[ Taylor,1991]

The loss function approach can be used to evaluate the effect of process improvement, the impact of tightening tolerances, or the effect of several inspection strategies in the total quality cost. The same can be said about maintenance costs.

Using the loss function we can determine the optimal control limits for parameters of the production process and check the intervals that minimise the total loss of production.

Taguchi (Taguchi,1989) considers the following methods to improve the parameters of the production, diagnosis, and adjustment processes so that the total loss is minimised. The effects of these methods in the overall manufacturing process efficiency can be assessed using the simulation model: the introduction of preventive maintenance measures; 
improvement of tools; improvement in the robustness of the process to achieve longer time between successive adjustments; the use of automatic devices in process diagnosis, process adjustment, and inspection; improvements in diagnosis and inspection methods as well as their location in the production process.

\section{CONCLUSIONS}

The loss function and simulation can be used to evaluate the impact of concrete improvements in the manufacturing performance. For these improvements to become a reality we have to develop and apply knowledge.

A closed loop system permits this knowledge to be continually added to and refined. This learning process is management's responsibility. Depending on management's abilities to enable this process to take place an incremental improvement in manufacturing performance will be achieve. This paper proposes a structure that supports the management of knowledge between all levels in an organisation.

Simulation models are being built to verify the approach. The case studies used are from discrete piece part manufacturing and the tile industry.

\section{References}

1-(Anderson, 1991) John C. Anderson, Kevin J. Dooley and Susan D.A. Misterek. The Role of Profound Knowledge in the Continual Improvement of Quality. Human Systems Management 10 (1991) 243-259.

2-(Deming, 1989).Deming, W.E. Comments regarding profound knowledge given at the Institute of Management sciences in Osaka, Japan, July 1989.

3-(Deming, 1986) Deming, W.E. Out of the Crisis. Massachusetts Institute of Technology, 1986.

4-(Edmondson, 1992) Harold E. Edmondson. Customer Satisfaction. In Manufacturing Systems: Foundations of World-Class Practice. Joseph A. Heim and W. Dale Compton, editors. National Academy Press. Washington, D.C. 1992.

5-(Kolarik, 1995) William J., Kolarik. Creating Quality. Concepts, Systems, Strategies, and Tools. McGrawHill International Editions. 1995.

6-(Liu, X, 1992) Xiaohe Liu. Process Knowledge Base for Continuous Process Improvement. PhD. Thesis. University of Minnesota. July 1992.

7-(Nakajima, 1988) Seiichi Nakajima. Introduction to Total Productive Maintenance Productivity Press, Inc. 1988.

8-(Shewhart, 1939). Shewhart, W.A., Statistical Method from the Viewpoint of Quality Control. The Graduate School of the Department of Agriculture, Washington D.C. 1939

9-(Simple ++, 1994). “Simple ++, Tutorial and Reference Manual. AESOP. 1994.

10-(Taguchi, 1986) Genichi Taguchi. Introduction to Quality Engineering. Asian Productivity Organization. 1986.

11-(Taguchi, 1989) Genichi Taguchi, Elsayed A. Elsayed, Thomas Hsiang. Quality Engineering in Production Systems. McGraw-Hill International Editions. 1989.

12-(Taguchi, 1993) Genichi Taguchi. Taguchi on Robust Technology Development. Bringing Quality Engineering Upstream. ASME Press. 1993.

13-(Taylor, 1991) Wayne A. Taylor. Optimization \& Variation Reduction in Quality McGraw-Hill 1991.

14-(Zeleny, 1989). Zeleny, M. Knowledge a New Form of Capital: Part 2. Knowledge Based Management Systems, Human Systems Management, vol 8, Number 2, 1989b, pp. 129-143. 